Document downloaded from:

http://hdl.handle.net/10251/36423

This paper must be cited as:

Cuenca Lacruz, AM.; García Gil, PJ.; Albertos Pérez, P.; Salt Llobregat, JJ. (2011). A nonuniform predictor-observer for a networked control system. International Journal of Control, Automation and Systems. 9(6):1194-1202. doi:10.1007/s12555-011-0621-5.

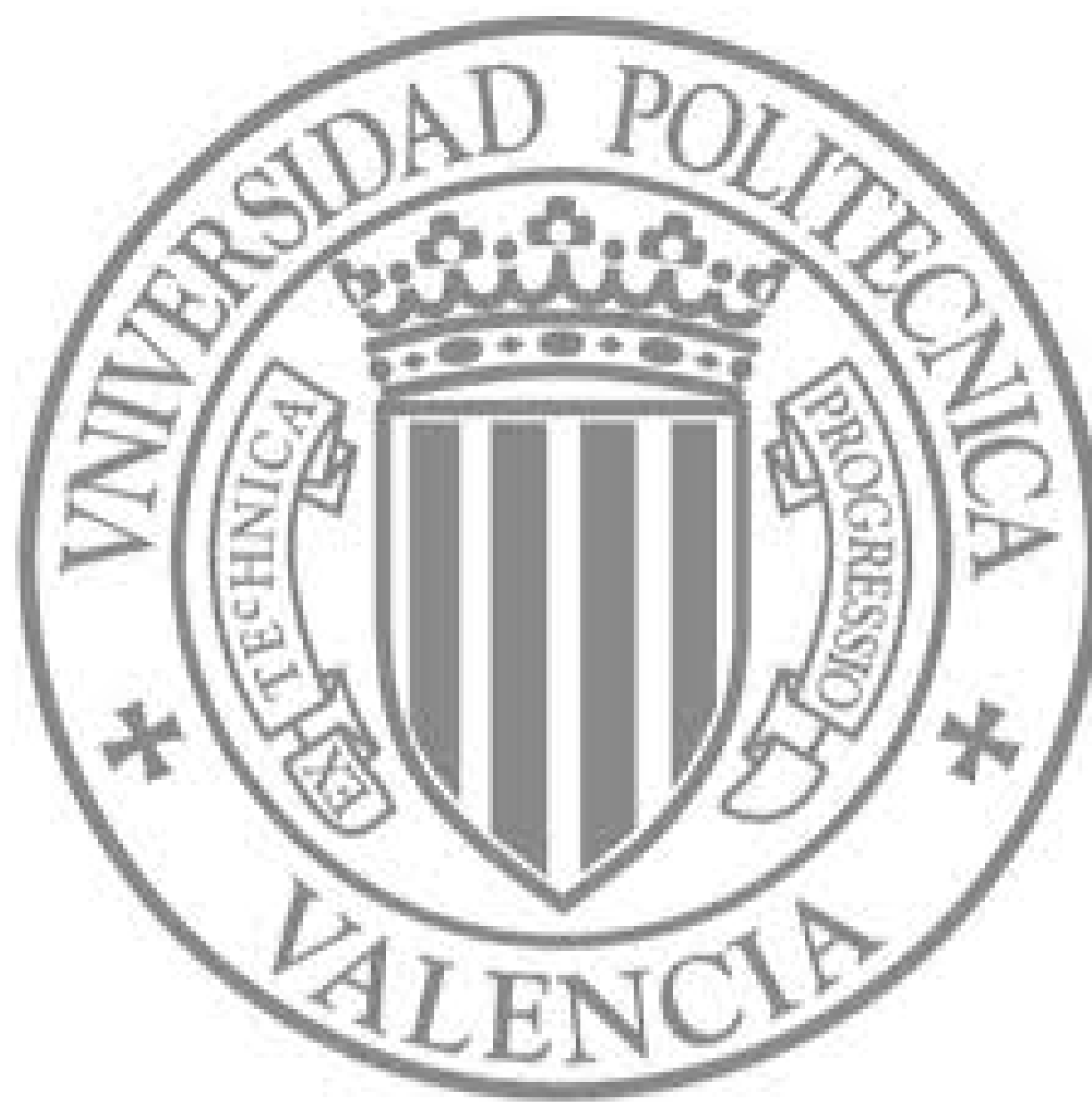

The final publication is available at

http://link.springer.com/article/10.1007\%2Fs12555-011-0621-5

Copyright Springer Verlag (Germany) 


\title{
A Non-Uniform Predictor-Observer for a Networked Control System
}

\author{
A. Cuenca, P. García, P. Albertos, and J. Salt
}

\begin{abstract}
This paper presents a Non-Uniform Predictor-Observer (NUPO) based control approach in order to deal with two of the main problems related to Networked Control Systems (NCS) or Sensor Networks (SN): time-varying delays and packet loss. In addition, if these delays are longer than the sampling period, the packet disordering phenomenon can appear. Due to these issues, a (scarce) nonuniform, delayed measurement signal could be received by the controller. But including the NUPO proposal in the control system, the delay will be compensated by the prediction stage, and the nonavailable data will be reconstructed by the observer stage. So, a delay-free, uniformly sampled controller design can be adopted. To ensure stability, the predictor must satisfy a feasibility problem based on a time-varying delay-dependent condition expressed in terms of Linear Matrix Inequalities (LMI). Some aspects like the relation between network delay and robustness/performance trade-off are empirically studied. A simulation example shows the benefits (robustness and control performance improvement) of the NUPO approach by comparison to another similar proposal.
\end{abstract}

Keywords: LMI, networked control system, network delay, packet disorder, packet loss, predictorobserver-based control.

\section{INTRODUCTION}

In conventional discrete-time control systems [1], the controller receives a uniform, not delayed input signal. From this signal, and only taking into account control requirements, a uniform control signal can be generated. Nevertheless, in NCS or SN [2] some problems appear due to sharing a communication medium among different devices (sensor, controller, actuator). Two of these problems are existence of time-varying delays and loss of data. In this context, the controller receives a nonuniform, delayed signal, which degrades control signal quality and hence control system performance. Then, in NCS not only control requirements but also this kind of issues must be faced. In the last years, many authors have introduced different solutions, for example: $H_{\infty}$ proposals [3-7], fuzzy methodologies [8], gain scheduling approaches $[9,10]$, together with adaptive predictors [11], dual-rate control strategies [12], packetbased transmission of several control signals $[13,14]$, impulsive time-delay feedback controllers [15], etc.

The present work addresses the problem of constructing control signals via estimation of the remote plant state. This state is based on measurements carried through a lossy network. This is an important problem not only treated in NCS but also in SN, remote sensing, and space exploration. Different solutions appear in [16-

This work was supported by the Spanish Ministerio de Ciencia y Tecnología Projects DPI2008-06737-C02-01 and DPI2009-14744C03-03, by Generalitat Valenciana Project GV/2010/018, by Universidad Politécnica de Valencia Project PAID06-08.

Angel Cuenca, Pedro García, Pedro Albertos and Julián Salt are with the Departamento de Ingeniería de Sistemas y Automática, Instituto Universitario de Automática e Informática Industrial, Universitat Politècnica de València, Camino de Vera s/n 46022 Valencia (Spain), (e-mails: \{acuenca,pggil,pedro,julian\}@isa.upv.es).
18] or in survey papers such as [19-21]. Some of them adopt Kalman filter based proposals to deal with the state estimation. Usually, it involves complex matrix calculations (incrementing computational load). However, in this work, a novel NUPO proposal is presented, which introduces basic operations (hence, low computational requirements are expected). Its main aim is to enable a uniformly sampled controller design (due to the observer inclusion), keeping the network-induced delay out of this design stage (as a result of the predictor consideration). Although the proposal can be used both for static and for dynamic controllers, this work is focused only on state feedback controllers in order to simplify the stability study.

The NUPO's prediction stage is defined by the number of steps for the ahead state prediction, $h$. In our proposal, $h$ is considered as a time-invariant parameter (the expected network delay). But the nature of the network delay is time-varying. Thus, two aspects must be studied: robust stability and performance degradation. To treat the first issue, the value $h$, in addition to the upper and lower network-induced delay bounds and the state feedback controller gain, are included in a time-varying delay-dependent condition, which must be solved in terms of LMI to ensure predictor stage stability ${ }^{1}$. Some authors consider both the controller and the observer gains in a same LMI feasibility problem (see, for example, $[23,24])$. However, it is remarkable that no one in the existing literature (to the best of the authors' knowledge) considers prediction parameters together with the controller gain in the same LMI feasibility problem. Regarding the second aspect (performance degradation), it is obvious that the closer to $h$ the

\footnotetext{
1 As the proposed predictor-observer structure holds the separation principle [22], separate stability of each block can be analyzed to prove the stability on the whole control scheme.
} 
network-induced delay is, the better control system performance can be obtained. Then, the goal is to quantify somehow this relation. Due to the high quantity of parameters that could be taken into account to obtain this expression (for example, plant to be controlled, sampling time, delay bounds, controller gain, etc), in this paper it will be empirically determined by means of an example. Finally, another important feature of the predictor is to be capable of working with possible unstable systems [22].

When the time-varying network delays are much longer than the sampling period, the packet disordering phenomenon can appear. This phenomenon may involve significant system degradation, since not updated information can be used to generate the control action. Some authors have studied this aspect, introducing solutions based basically on robust control [25] and predictive control [26]. In the present work, due to the observer consideration, a straightforward solution based on a simple comparison carried out in a Measurement Selector (MS) can be adopted. So, using time-stamping techniques when a measurement arrives to the MS, this measurement will be actually taken if it is newer than the last one taken. Otherwise, the arriving measurement is discarded (as being a packet dropout) and it must be observed.

In this work a harsh environment is assumed in such a way that only few samples (due to packet loss) will finally arrive to the controller. However, this proposal is easily adaptable to an event-based control approach [27], where an event detector can be located at the system output. So, when certain threshold is passed by this output, an event is triggered in order to send the sample through the network to the controller. In [28], this approach is considered.

In conclusion, the significance of the present work lies in the next issues:

- the inclusion of prediction parameters together with the controller gain in the same LMI feasibility problem,

- to adopt a straightforward solution for the state estimation as a consequence of considering the MS together with the observer,

- the introduction of an empirical expression to easily evaluate the relation between the network delay and the robustness/performance trade-off,

- to extend previous studies developed by the authors in [10] and [11], where neither packet dropouts nor packet disordering are faced.

Regarding the last aspect, a multi-rate networked control structure is considered in [10] and [11], where the system output is measured at slow period, and the control action is updated at fast period. In both works the main assumptions are: the time-varying network delays are less than a slow (measurement) period, and there are no packet dropouts. Thus, no packet disordering can appear.

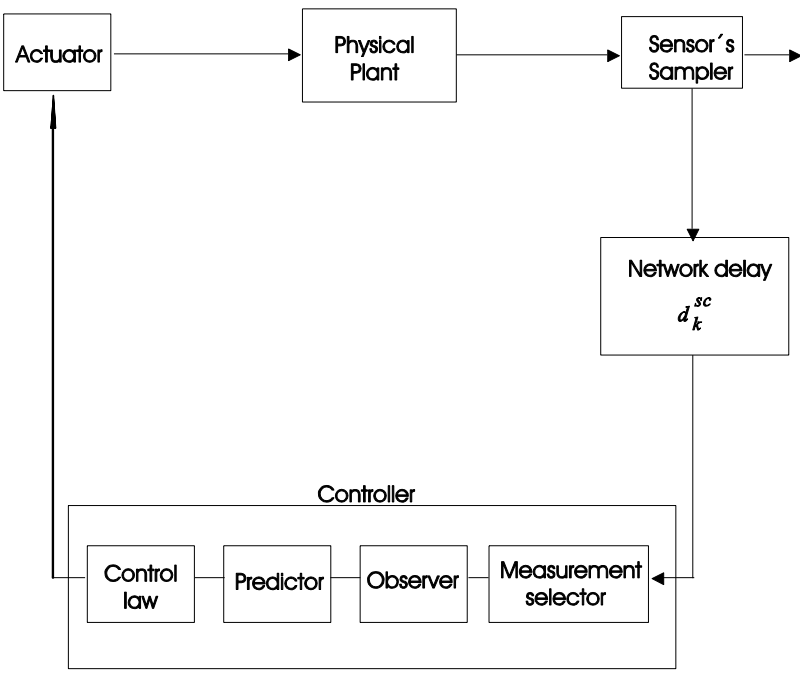

Fig. 1. NCS or SN configuration.

The paper is organized as follows: in section 2, the problem scenario considering a discrete-time framework is presented. In section 3 the NUPO proposal is introduced. Robust stability aspects for the predictor are expressed in terms of LMIs, and design steps for the observer are defined. Section 4 presents two examples: in the first one, an empirical relation between network delay and control performance is established, and in the second example, both an improvement of the control performance and a higher degree of robustness are achieved by our approach when comparing to another example illustrated in [29]. Finally, section 5 enumerates the main conclusions of the work.

\section{PROBLEM SCENARIO}

\subsection{Network setup and network-induced delay}

Let us consider the NCS illustrated in Fig. 1. The network setup under consideration is a one-channel feedback NCS, that is, a NCS where a remote sensor sends its information through a network to a controller collocated with the actuator ${ }^{2}$. In this work, the network devices are considered time-triggered. In the controller four different stages are included: measurement selection, state estimation, state prediction, and the control action generation. As it will be later discussed, the first stage treats the packet disorder phenomena, the second stage deals with the packet loss, and the third stage compensates for the network delay.

In this NCS, the physical plant can be modeled by the following discrete-time linear system

$$
\begin{aligned}
& x_{k+1}=A x_{k}+B u_{k}, \\
& y_{k}=C x_{k-d_{k}^{s c}},
\end{aligned}
$$

where $x_{k} \in \mathfrak{R}^{n}$ is the state vector, $u_{k} \in \mathfrak{R}^{p}$ is the control input, $y_{k} \in \mathfrak{R}^{q}$ is the output. $A, B, C$, are system

2 This network setup has been commonly used to investigate the effects of sampling and delay in the stability of NCS [20]. 
matrices with the appropriate dimensions, and $d_{k}^{s c} \in \aleph$ is the time-varying sensor-to-controller network delay. The computation delay (if it exists) is considered negligible or lumped together under the previous delay ${ }^{3}$. Let us assume $T$ as a uniform sampling period in such a way that the sampling time instant $t_{k}=k T$ (where $k \in \aleph$ and $T>0$ ). As the main interest of this work is to achieve a stabilizing controller, disturbances or measurement noises are not considered.

Using synchronization protocols and time-stamping techniques, the current sensor-to-controller delay $d_{k}^{s c}$ can be measured. So, with this current value, the problems regarding packet disorder and state estimation can be faced (more information below). However, with respect to the compensation step, as the predictor works with a time-invariant delay value $h$ (more details in section 3), the fact of knowing the current value of $d_{k}^{s c}$ is not relevant. Otherwise, it is more interesting to know a priori, statistical information about the delay (for example, distribution function, lower and upper bounds, etc). So, from this information, the network delay can be expressed as $d_{1} \leq d_{k}^{s c} \leq d_{2}$, being $d_{1}$ the lower bound and $d_{2}$ the upper bound. On the following $d_{k}^{s c}$ will be denoted with shorthand $d_{k}$ if no confusion arises from the context.

In this work, for brevity, neither small uncertainty in the knowledge of the delay $d_{k}$ nor in the sampling period $T$ are considered (see in [22] a related study where $d_{k}$ is considered a time-invariant delay).

\subsection{State feedback controller}

As commented, a state feedback controller will be considered, being its control law

$$
u_{k}=-K x_{k+d_{k}}
$$

which provides the required behavior of the closed loop system (1)-(3), characterized by the system matrix (A$B K$ ). But, some problems arise (to be treated in detail in section 3):

- Due to the network-induced delay, the state $x_{k+d_{k}}$ is not yet known when generating $u_{k}$. So, a state predictor is required (considering that (1)-(2) could be unstable).

- Due to the packet loss and time-varying delays, the

\footnotetext{
3 As a one-channel feedback setup is considered, the controller-toactuator delay does not appear. While the sensor-to-controller delay can be known at the controller device and considered to calculate the current control action, the controller-to-actuator delay (if considered) is not known at this time; therefore, no exact correction for this delay can be made in this moment, requiring some control techniques to deal with it ( $H_{\infty}$ proposals, fuzzy methodologies, gain scheduling approaches, etc; see some references at the beginning of section 1).
}

output measurement $\tilde{y}_{k}$ (to be defined in (4)) is not available every $k^{\text {th }}$ sampling instant. Thus, an observer will be included to estimate the nonavailable data.

\subsection{Packet loss}

In order to model the packet loss process, this expression is used

$$
\tilde{y}_{k}=\theta_{k} y_{k}
$$

where $\theta_{k}$ takes values in $\{0,1\}$ regarding the packet dropouts $^{4}$. As commented, due to these dropouts and the time-varying delays, the observer receives a (scarce) non-uniform signal $\tilde{y}_{k}$ to be uniformly reconstructed (at period $T$ ). By implementing time-stamping techniques, the number of $T$-periods between two consecutive available measurements (the previous one taken in time, say, $k_{\text {prev }}$, and the current one taken in time k) can be determined and defined as $N_{k}$

$$
1 \leq N_{k} \leq \bar{N}
$$

where $\tilde{N}=\bar{N}-1$ is the consecutive non-available packet upper-bound (from the observer point of view, $\tilde{N}$ is the actual upper-bound for packet dropouts; the example in Fig. 2 illustrates this aspect).

\subsection{Measurement Selector}

Finally, as the network induced delays can be much longer than the sampling period $T$ and, in addition, they are time-varying, the packet disordering phenomenon can appear. Due to the subsequent use of the observer, this problem can be easily solved by computing a simple comparison. This logic operation is carried out by the Measurement Selector (MS) and requires the timestamping information. This is the MS operation mode:

- the sensor adds a time-stamp to the sent packet, say $t s\left(y_{k}\right)$,

- when an available measurement $\tilde{y}_{k} \quad\left(\right.$ with $\left.\theta_{k}=1\right)$ arrives to the MS (in time $k$ ), it will be actually taken if it is newer than the last one taken (in time $k_{\text {prev }}$ ), that is, if $t s\left(y_{k}\right) \equiv t s\left(\tilde{y}_{k}\right)>t s\left(\tilde{y}_{k \text { prev }}\right)$.

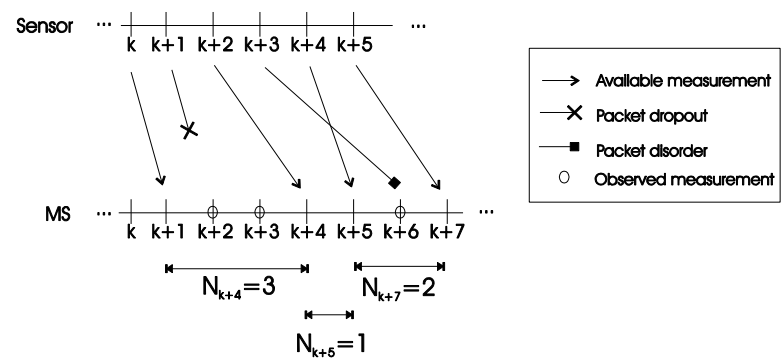

Fig. 2. Measurement Selector operation mode (example).

\footnotetext{
${ }^{4}$ In this work, the packet dropout process is defined as a totally random (but bounded) sequence, but it could be defined, for example, as a Bernoulli sequence [30].
} 


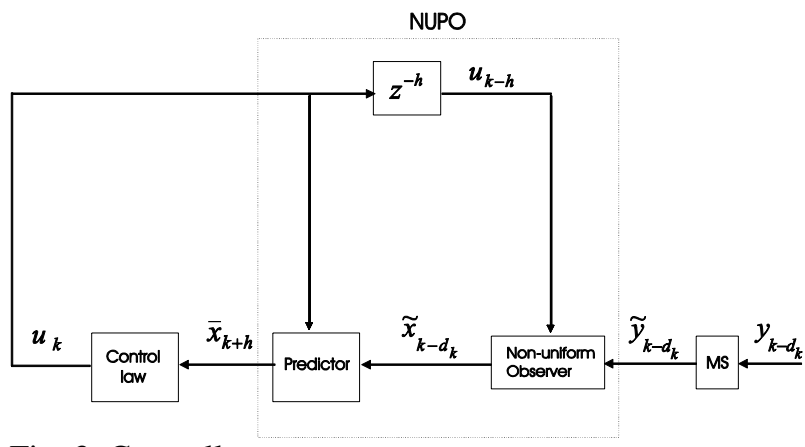

Fig. 3. Controller.

- otherwise, the arriving measurement is discarded (treated like a packet dropout, that is, $\tilde{y}_{k}$ with $\theta_{k}=0$ ), so $N_{k}$ is incremented and the current measurement must be observed.

In Fig. 2, an example to show how the MS works is illustrated.

\section{NON-UNIFORM PREDICTOR-OBSERVER (NUPO) PROPOSAL}

Fig. 3 shows a detailed scheme of the controller, where the predictor-observer is included. Next, predictor and observer stages are separately defined.

3.1. Predictor stage: Time-varying delay-dependent stability condition

As commented, in order to design the proposed predictor, it is absolutely necessary to determine the number of steps for the ahead state prediction, $h \in \aleph^{+}$. The predictor uses this value as a time-invariant parameter (the expected delay). This fact can make easier the predictor design (for example, it can be defined offline), but it can suppose a degradation of the control system performance (the higher $\Delta h=\left|h-d_{k}\right|$ is, the worse control system performance can be experimented). To determine $h$, a priori information about the parameter $d_{k}$ (that is, its mean, median, mode, etc) must be studied. Once the value for $h$ is determined, two goals must be reached:

- Fist of all, to ensure robust stability. From the lower $d_{1}$ and upper $d_{2}$ bounds, predictor stability must be guaranteed for this $h$ and taking into account the desired controller gain $K$. In order to reach this goal, a LMI feasibility problem must be solved.

- Secondly, to determine the relation between $\Delta h$ and performance degradation. This relation depends on different control system parameters, and our proposal is to be empirically studied.

So, achieving both goals, the robustness/performance trade-off can be evaluated. In section 4 , an example will show these aspects.

Regarding the first goal, a perfectly known process model is considered (1)-(2), yielding the state feedback control law

$$
u_{k}=-K \bar{x}_{k+h},
$$

where $K \in \mathfrak{R}^{m \times n}$, and $\bar{x}_{k+h}$ is the next $h$-step ahead state prediction law, which takes the form

$$
\bar{x}_{k+h}=A^{h} \tilde{x}_{k-d_{k}}+A^{h-1} B u_{k-h}+\cdots+B u_{k-1},
$$

being $\tilde{x}_{k-d_{k}}$ the delayed state estimation, and $u_{k-i}$ $(i=1, \ldots, h)$ the past control actions.

Treating $\Delta h$ as a delay uncertainty, a sufficient stability condition can be proposed in order to ensure a maximum $\Delta h$. So, from (1)-(2), (6)-(7), the closed-loop system state yields

$$
x_{k+1}=(A-B K) x_{k}-B K A^{h} \tilde{x}_{k-d_{k}}+B K A^{h} x_{k-h},
$$

then, (8) is asymptotically stable for any $d_{1} \leq d_{k} \leq d_{2}$, if there exist positive definite matrices $P, Q_{1}, Q_{2}, Z_{1}$ and $Z_{2}$, and matrices $X_{1}, X_{2}, Y_{1}$ and $Y_{2}$, such that the following LMI constraints hold (see [31] for details, where a similar case is studied)

$$
\begin{aligned}
& \left(\begin{array}{cccccc}
\Gamma & -Y_{1} & -Y_{2} & M^{\mathrm{T}} P & d_{2}(M-I)^{\mathrm{T}} Z_{1} & h(M-I)^{\mathrm{T}} Z_{2} \\
* & -Q_{1} & 0 & A_{1}^{\mathrm{T}} P & d_{2} A_{1}^{\mathrm{T}} Z_{1} & h A_{1}^{\mathrm{T}} Z_{2} \\
* & * & -Q_{2} & -A_{1}^{\mathrm{T}} P & -d_{2} A_{1}^{\mathrm{T}} Z_{1} & -h A_{1}^{\mathrm{T}} Z_{2} \\
* & * & * & -P & 0 & 0 \\
* & * & * & * & -d_{2} Z_{1} & 0 \\
* & * & * & * & * & -h Z_{2}
\end{array}\right)<0, \\
& \left(\begin{array}{ll}
X_{1} & Y_{1} \\
Y_{1}^{\mathrm{T}} & Z_{1}
\end{array}\right) \geq 0, \quad\left(\begin{array}{cc}
X_{2} & Y_{2} \\
Y_{2}^{\mathrm{T}} & Z_{2}
\end{array}\right) \geq 0,
\end{aligned}
$$

where $M:=(A-B K), A_{1}:=B K A^{h}$, and $\Gamma:=-P+d_{2} X_{1}+$ $h X_{2}+Y_{1}+Y_{1}{ }^{\mathrm{T}}+Y_{2}+Y_{2}^{\mathrm{T}}+\left(d_{2}-d_{1}+1\right) Q_{1}+Q_{2}$. As known in LMI expressions, '*' represents a term induced by symmetry. The superindex ' $T$ ' denotes the matrix transpose.

Remark 1: This is a standard LMI expression, which can be solved in few iterations by widely known LMI solvers (for example, [32]).

\subsection{Non-uniform observer stage}

To estimate the system state, different kind of observers can be used: a Kalman filter [20, 27], a logconcave observer [33], etc. In this work, due to its straightforward design, a non-uniform observer is considered. This observer receives two delayed signals (the non-uniform output signal and the control signal), and generates a uniform state estimation (at period T). So, under normal operation, the state vector is estimated through the observer

$$
\tilde{x}_{k-d_{k}+1}=A \tilde{x}_{k-d_{k}}+B u_{k-h}+L\left(\tilde{y}_{k-d_{k}}-C \tilde{x}_{k-d_{k}}\right) \text {, }
$$

where the observer matrix is $A_{L}=A-L C$.

In the case of outputs $\tilde{y}_{k}$ being received in a nonuniform way by the observer, and taking into account the relation $N_{k}$ between two consecutive available 
measurements, the new observer will be:

$$
\tilde{x}_{k-d_{k}+1}=A \tilde{x}_{k-d_{k}}+B u_{k-h}+\tilde{L}\left(\tilde{y}_{k-d_{k}}-C \tilde{x}_{k-d_{k}}\right),
$$

where $\tilde{L}$ is chosen such that

$$
A^{N_{k}}-\tilde{L} C A^{N_{k}-1}
$$

is Schur $^{5}$. Note, $\tilde{L}$ will be null if there is no measurement of the output.

Proof: The delayed state estimation in time $k+N_{k}$ yields

$$
\begin{aligned}
\tilde{x}_{k-d_{k}+N_{k}}=A^{N} \tilde{x}_{k-d_{k}}+W_{u, k-h}+ \\
+\tilde{L} A^{N_{k}-1}\left(\tilde{y}_{k-d_{k}}-C \tilde{x}_{k-d_{k}}\right),
\end{aligned}
$$

where

$$
\begin{aligned}
W_{u, k-h}= & B u_{k-h+N_{k}-1}+B A u_{k-h+N_{k}-2}+ \\
& +\cdots+B A^{N_{k}-1} u_{k-h},
\end{aligned}
$$

So, the non-uniform observer matrix is $A_{\tilde{L}}=A^{N_{k}}-\tilde{L} C A^{N_{k}-1}$. As known, if the pair $\left(A^{N_{k}}, C A^{N_{k}-1}\right)$ is detectable, then $\tilde{L}$ can be chosen to make $A_{\tilde{L}}$ Schur. In this way, the state estimation error $e_{k}$ will tend to 0 , since

$$
\begin{aligned}
& e_{k}=x_{k}-\tilde{x}_{k}, \\
& e_{k+N_{k}}=x_{k+N_{k}}-\tilde{x}_{k+N_{k}}=\left(A^{N_{k}}-\tilde{L} C A^{N_{k}-1}\right) e_{k} .
\end{aligned}
$$

A similar approach is presented in [34], but there, a uniform, non-delayed observer input signal is considered.

Remark 2: Although calculating computational costs is not the goal of this paper, it is interesting to note that, both in the state estimation and in the state prediction, basic operations are required. Thus, if the NUPO approach were compared to other existing solutions in the literature (for example, those based on the Kalman filter; see in [20]), lower computational load (and easier implementation) would be expected.

\section{SIMULATION EXAMPLES}

In this section two examples are presented. In the first one, a complete study is developed for a double integrator plant in order to finally obtain an empirical relation between network delay and control performance, which can be used to evaluate the robustness /performance trade-off. In the second one, the NUPO proposal is compared to the approach presented in [29] in order to observe both the robustness benefits and the control performance improvement which are capable to reach our approach.

\footnotetext{
5 Schur: the eigenvalue are inside unit circle.
}

\subsection{Robust stability vs. performance}

To achieve the final goal of this example, that is, to evaluate the relation among network delay, performance and robust stability, a detailed study about how to design the NUPO based control system is presented. Several steps must be carried out:

- First of all, to define the main parameters of the network-induced delay. So, using a priori information about the network behavior, some aspects like lower and upper bounds, delay distribution function, etc, can be determined.

- Secondly, and depending on the previous parameters, to determine $h$ for the predictor design.

- Thirdly, to establish the desired control performance (based on some control index) in order to design the state feedback controller gain. In addition, the nonuniform observer gain must be determined.

- Fourthly, to ensure robust stability for the considered NCS by means of the proposed LMI (9).

- Finally, to quantify how control performance is degraded when incrementing $\Delta h=\left|h-d_{k}\right|$.

A realisation of the double integrator $1 / s^{2}$ at period $T=0.05 \mathrm{~s}$ is obtained as

$$
\begin{aligned}
& x_{k+1}=\left(\begin{array}{cc}
1 & 0 \\
0.05 & 1
\end{array}\right) x_{k}+\left(\begin{array}{c}
0.05 \\
0.0013
\end{array}\right) u_{k}, \\
& y_{k}=\left(\begin{array}{ll}
0 & 1
\end{array}\right) x_{k} .
\end{aligned}
$$

Let us assume that the control system is implemented over an IP network. In this case, it is usual to approximate IP network delays as a generalized exponential distribution [9], following this expression

$$
P\left[d_{k}\right]= \begin{cases}\frac{1}{\phi} e^{\frac{-\left(d_{k}-\eta\right)}{\phi}}, & d_{k} \geq \eta, \\ 0, & d_{k}<\eta\end{cases}
$$

where, in our example, $\phi=\eta=0.1$. As commented in section 3.1, several values are candidate to determine $h$. Let us use in this case the peak of $P\left[d_{k}\right]$, since both the majority of network-induced delays and the median value are close to it [9]. So, $h=0.1$.

In this case, as index to evaluate the control performance, the settling time is taken. Then, if the closed-loop poles are desired to be located in $s=-2$ and $s=-1$, the next state feedback controller gain is obtained: $K=[3,2]$. For simplicity, the observer is designed with a uniform dropout pattern $N_{k}=4$, and locating its poles in $z^{N_{k}}=0.19^{4}$ and $z^{N_{k}}=0.15^{4}$. Consequently, the observer gain is $\tilde{L}=[13.77,1.66]^{\mathrm{T}}$.

Now, from $K$ and $h$ the LMI is used to determine the lower $d_{1}$ and upper $d_{2}$ bounds that ensure robust stability. So, $d_{1}=0.1$ and $d_{2}=0.2$. Note that the LMI provides only a stability sufficient condition, and hence the obtained bounds can be conservative. This fact can be easily checked by simulation (as shown in Fig. 4), where the system is on the verge of instability when $d_{2}=0.5$. However, the upper bound provided by the LMI could be 
understood as a value from which control performance starts to be degraded. In this figure, and the next ones, circles on the time axes indicate the time instants when measurements are available (no packet loss). In this case, as $N_{k}$ is invariant, a uniform pattern is observed.

Let us relax the control requirements, locating now the closed-loop poles in $s=-2, s=-0.5$. A new $K=[2.5,1]$ is obtained, and consequently the LMI provides a wider range $\left(d_{1}=0.1, \quad d_{2}=0.3\right)$. Then, as expected, the robustness/performance trade-off follows this law: the higher control performance is required, the worse robustness is achieved. As previously, by simulation (see Fig. 5), the upper bound can be incremented up to $d_{2}=0.60$. But, from the value provided by the LMI $\left(d_{2}=0.3\right)$, the control performance worsens.

Fig. 6 summarizes the control performance degradation (in \% of the settling time, say $P D(\%)$ ) experimented when $\Delta h$ is more and more incremented. This relation can be approximated by means of a Gaussian-like expression in this way

$$
P D(\Delta h)(\%)=e^{-\frac{\Delta h^{9}}{2\left(w \cdot d_{2}\right)^{9}}} \cdot 100,
$$

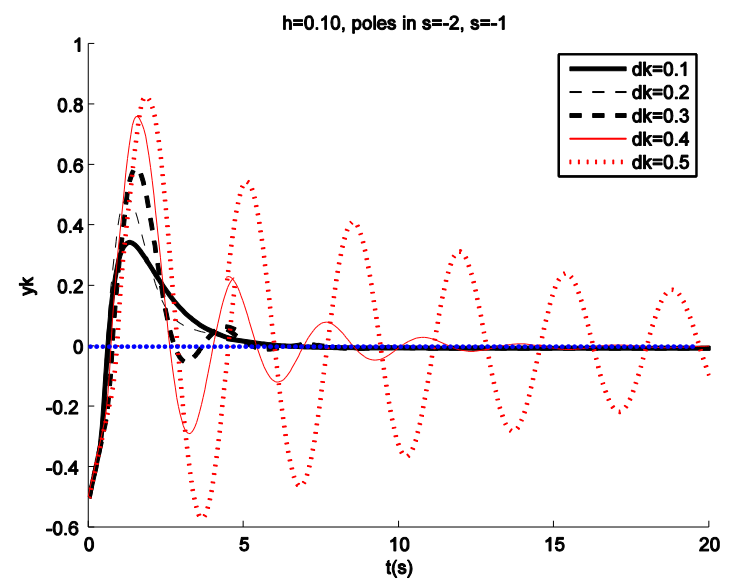

Fig. 4. System output, poles in $s=-2, s=-1$.

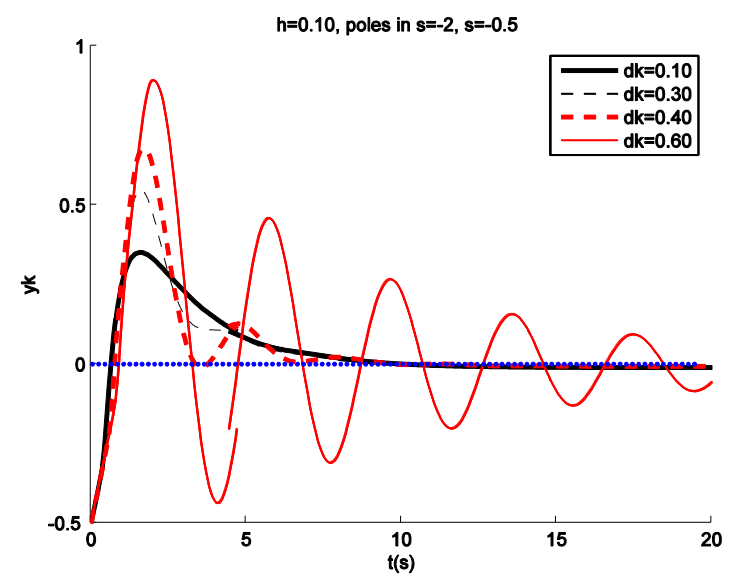

Fig. 5. System output, poles in $s=-2, s=-0.5$.

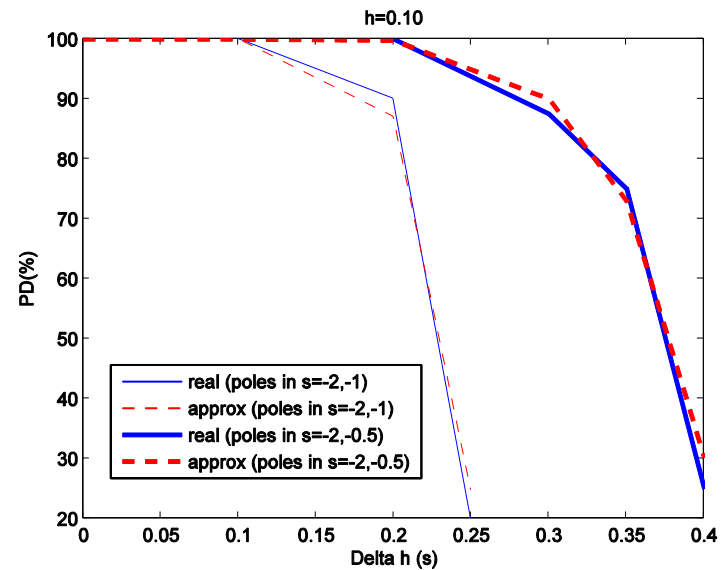

Fig. 6. Empirical relation: network delay vs. control performance.

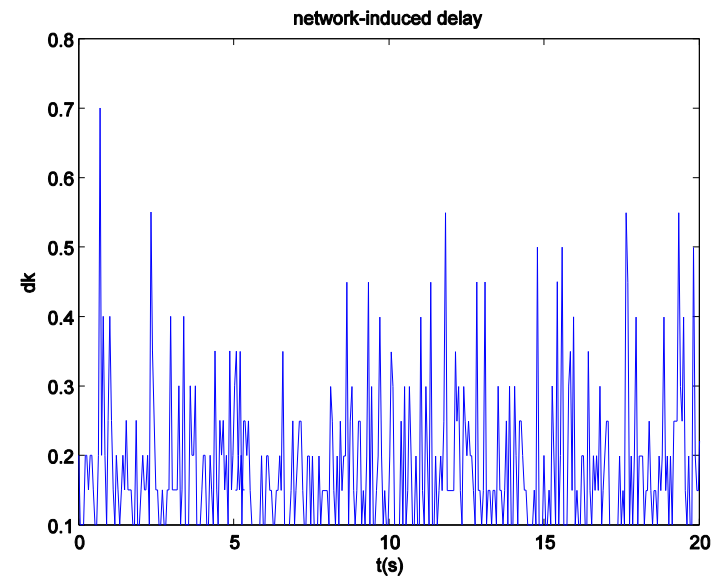

Fig. 7. Network delay pattern.

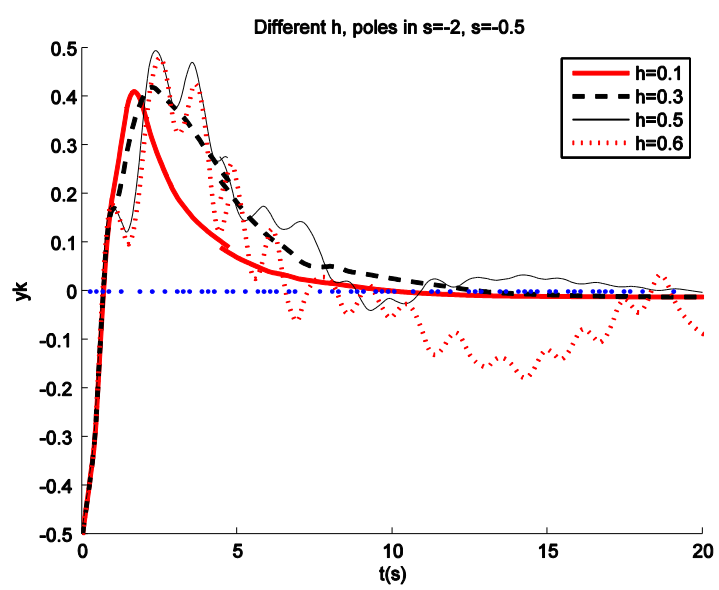

Fig. 8. System output for different $h$.

where $d_{2}$ is the value provided by the LMI condition, and $w$ is a weighting coefficient (in this case, $w=1.1$ ). The approximation exhibits a maximum error of $\pm 3 \%$. Note that the control performance degradation appears when considering higher delay values, which are less probable in this distribution (19).

Finally, the next study chooses different $h$ in order to observe their influence in the control performance when 
the time-varying delays $d_{k}$ follow the delay distribution defined by (19). So, considering a determined delay pattern (shown in Fig. 7) and, for example, the case where the relaxed performance is required, Fig. 8 shows the consequent results. As expected, the higher $h$ is taken, the more degraded the control system performance will be, since, according to the delay pattern (Fig. 7), the majority of delays $d_{k}$ are low values and hence, a higher $h$ means a higher $\Delta h$. Note that now, despite having again $N_{k}=4$, circles on the time axes indicate a nonuniform measurement pattern. This is due to appearing the packet disordering phenomenon, which discards disordered samples.

\subsection{Comparison}

The main aim of this comparison is to show that, despite delay variations, whereas the NUPO proposal keeps control performance and stability, the approach used in [29] is on the verge of instability.

The continuous system to be studied is

$$
\dot{x}(t)=\left(\begin{array}{ccc}
-1 & 0 & -0.5 \\
1 & -0.5 & 0 \\
0 & 0 & 0.5
\end{array}\right) x(t)+\left(\begin{array}{l}
0 \\
0 \\
1
\end{array}\right) u(t) .
$$

In [29] several cases are studied for this system. The last one considers a sampling period $T=0.5 \mathrm{~s}$, an arbitrary packet loss with the upper-bound $\tilde{N}=2$ (then $1 \leq N_{k} \leq 3$ ), and a time-invariant network delay $d_{k}=1$. So, in that work the next state feedback control law is designed: $u_{k}=[-0.0109,-0.0074,-0.7175] x_{k}$.

Considering the same design parameters for the NUPO proposal, when locating the continuous closed-loop poles in $S=[-0.886,-0.885,-0.884]$ the next state feedback gain is obtained: $K=[-0.1347,-0.0658,1.291]$. With this $K$ and $h=d_{k}=d_{1}=d_{2}$, (9) is satisfied, hence predictor stability is reached. Under normal operation, if the observer (discrete) poles are located in $z=[0.581,0.582$, $0.583]$, the observer gain yields: $L=[0.505,0.418$, $-1.516]^{\mathrm{T}}$. Then, depending on $N_{k}, \tilde{L}$ (the non-uniform observer gain) will be obtained when locating the poles in $z^{N_{k}}$, achieving stability for (12).

The closed-loop control system simulation comparing both approaches is depicted in Fig. 9, taking the initial state as $x_{0}=[-5,0,5]^{\mathrm{T}}$. The NUPO proposal achieves a fast convergence rate (up to a $30 \%$ faster) than the control method in [29]. Circles on the time axes indicate the time instants when measurements are available (no packet loss). This control performance improvement is achieved despite generating a similar (in magnitude) control action signal (see Fig. 10). Nevertheless, it is worth to note that a strong control action is applied in the initial step. This can be reduced but the transient response will be degraded.

Finally, Fig.11 shows how robust the NUPO design is compared to [29]. So, under the assumption of an eventual increment in the network-induced delay (for example, now $d_{k}=2$ ), the NUPO proposal is able to keep system stability using the previous $h=1$ and the previous controller and observer gains, and generating a control signal (see Fig.12) which is similar to that shown in Fig. 10 (hence, control performance is hardly degraded). Nevertheless, the system state for the control method in [29] is on the verge of instability, since a new controller gain must be designed for the new delay.

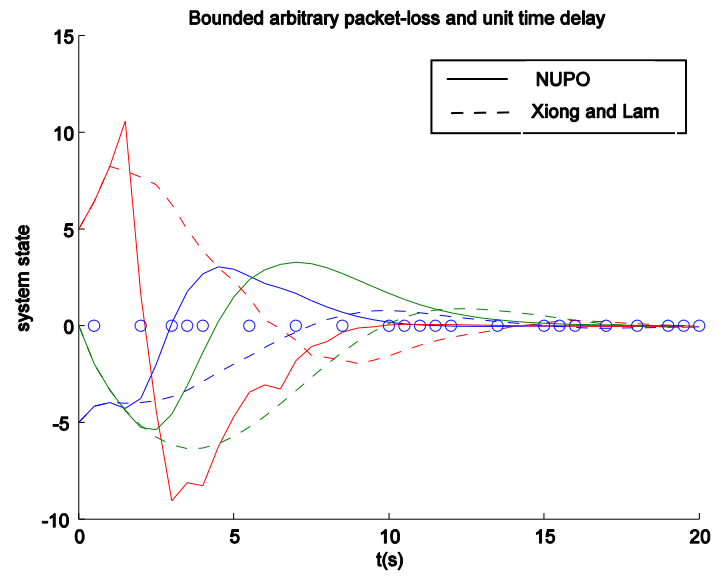

Fig. 9. System state comparison with a unit delay.

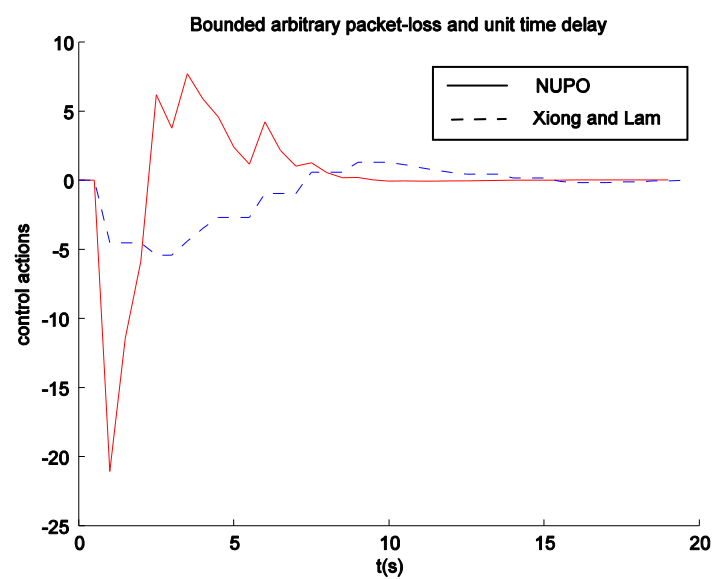

Fig. 10. Control action comparison with a unit delay.

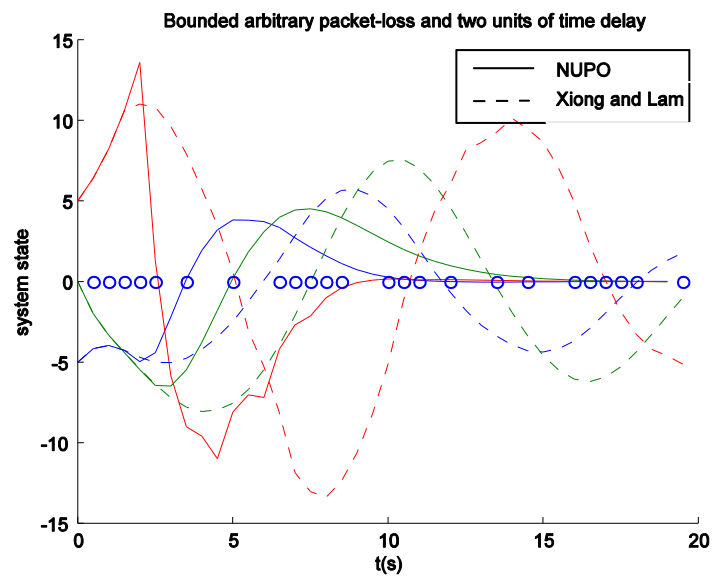

Fig. 11. System state comparison with two units of delay. 


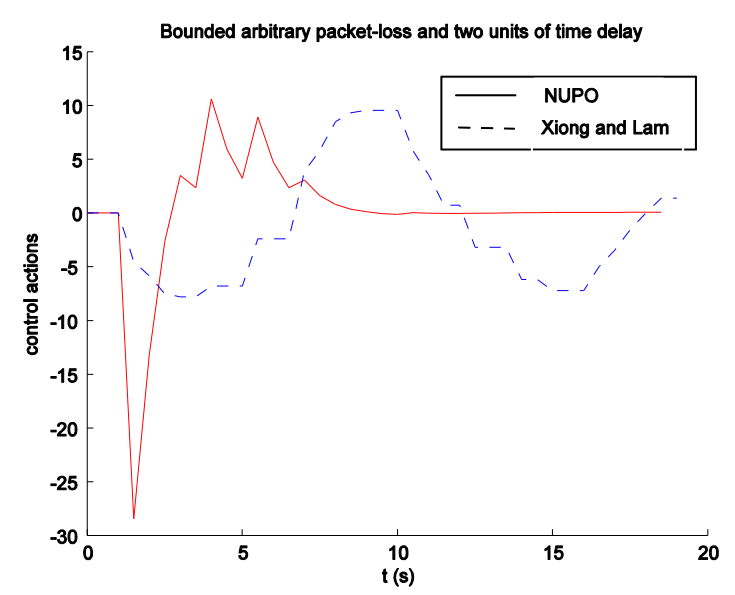

Fig. 12. Control action comparison with two units of delay.

\section{CONCLUSIONS}

Time-varying network delays and packet loss are two of the most important problems that appear in a NCS or SN. The packet disordering phenomenon can also appear, if time-varying delays are longer than the sampling period. In order to deal with these aspects, in this work a Non-Uniform Predictor-Observer (NUPO) based control is proposed. The NUPO approach involves basic operations, and hence, low computational requirements are expected. Due to the aforementioned issues, the measurement signal can become a disordered, non-uniformly sampled signal. Then, firstly, a Measurement Selector is required to select only the updated information. Secondly, the Non-Uniform Observer is needed to reconstruct the non-available output data. Finally, the prediction stage, which is capable to work with a possible unstable system, put the network-induced delay out of the control loop. Thus, with the NUPO proposal, the controller can generate a uniform, delay-free control signal.

Since the separation principle holds in this control approach, separate stability can be analyzed for predictor and observer. In the first case, a time-varying delaydependent stability condition in terms of LMIs must be satisfied. In this LMI condition, controller and predictor parameters are both included. This fact has been found in no work in the related literature (to the best of authors' knowledge).

Due to the predictor operation mode, some performance degradation can be experimented when the network delay is different to the expected one. Thus, it is interesting to quantify the relation among network delay, control performance and robustness. A simulation example determines empirically this relation. Another example compares our proposal to another similar one, and shows some benefits of the NUPO proposal like control performance improvement and robustness.

\section{REFERENCES}

[1] K. Ogata, Discrete-time control systems. PrenticeHall, Inc. Upper Saddle River, NJ, USA, 1987.
[2] Y. Tipsuwan and M. Chow, "Control methodologies in networked control systems," Control Eng. Practice, vol. 11, no. 10, pp. 1099-1111, 2003.

[3] T. Jia, Y. Niu, and X.Wang, " $H \infty$ control for networked systems with data packet dropout," Int. J. Control, Autom., and Syst., vol. 8, no. 2, pp. 198203, 2010.

[4] Y. Wang and G. Yang, "Robust $H \infty$ model reference tracking control for networked control systems with communication constraints," Int. J. Control, Autom., and Syst., vol. 7, no. 6, pp. 992-1000, 2009.

[5] H. Gao and T. Chen, "Network-based Hळo output tracking control," IEEE Trans. Autom. Control, vol. 53, no. 3, pp. 655-667, 2008.

[6] H. Karimi, "Robust $H \infty$ filter design for uncertain linear systems over network with network-induced delays and output quantization," Modeling, Identification and Control, vol. 30, no. 1, pp. 2737, 2009.

[7] —, "Delay-range-dependent linear matrix inequality approach to quantized $H \propto$ control of linear systems with network-induced delays and norm-bounded uncertainties," Proc. of the Inst. of Mech. Eng., Part I: J. of Syst. and Control Eng., vol. 224, no. 6, pp. 689-700, 2010.

[8] K. Lee, S. Lee, and M. Lee, "Remote fuzzy logic control of networked control system via ProfibusDP," IEEE Trans. Ind. Electron., vol. 50, no. 4, pp. 784-792, 2003.

[9] Y. Tipsuwan and M-Y. Chow, "Gain scheduler middleware: a methodology to enable existing controllers for networked control and teleoperationPart I: Networked Control," IEEE Trans. on Industrial Electronics, vol. 51, no. 6, pp. 1218-1227, December 2004.

[10] A. Sala, A. Cuenca, and J. Salt, "A retunable PID multi-rate controller for a networked control system," Inform. Sci., vol. 179, no. 14, pp. 23902402, June 2009.

[11] A. Cuenca, J. Salt, V. Casanova, and R. Pizá, “An approach based on an adaptive multi-rate Smith predictor and gain scheduling for a networked control system: implementation over Profibus-DP," Int. J. Control, Autom., and Syst., vol. 8, no. 2, pp. 473-481, April 2010.

[12] A. Cuenca, J. Salt, A. Sala, and R. Pizá, “A delaydependent dual-rate PID controller over an Ethernet network," IEEE Trans. Ind. Informat., vol. 7, no. 1, pp. 18-29, Feb. 2011.

[13] Y. Tian and D. Levy, "Compensation for control packet dropout in networked control systems," Inform. Sci., vol. 178, no. 5, pp. 1263-1278, 2008.

[14] Y. Zhao, G. Liu, and D. Rees, "Modeling and stabilization of continuous-time packet-based networked control systems." IEEE Trans. Syst., Man, Cybern. B, vol. 39, no. 6, pp. 1646-1652, Dec. 2009

[15] X. Zhao, S. Fei, and C. Sun, "Impulsive controller 
design for singular networked control systems with packet dropouts," Int. J. Control, Autom., and Syst., vol. 7, no. 6, pp. 1020-1025, 2009.

[16] H. Gao and T. Chen, " $H \infty$ estimation for uncertain systems with limited communication capacity," IEEE Trans. Autom. Control, vol. 52, no. 11, pp. 2070-2084, 2007.

[17] S. Oh, L. Schenato, P. Chen, and S. Sastry, "Tracking and coordination ofmultiple agents using sensor networks: System design, algorithms and experiments," Proc. of the IEEE, vol. 95, no. 1, pp. 234-254, 2007.

[18] M. Moayedi, Y. Foo, and Y. Soh, "Optimal and suboptimal minimum-variance filtering in networked systems with mixed uncertainties of random sensor delays, packet dropouts and missing measurements," Int. J. Control, Autom., and Syst., vol. 8, no. 6, pp. 1179-1188, 2010.

[19] W. Zhang, M. Branicky, and S. Phillips, "Stability of networked control systems," IEEE Control Syst. Mag., vol. 21, no. 1, pp. 84-99, 2001.

[20] J. Hespanha, P. Naghshtabrizi, and Y. Xu, "A survey of recent results in networked control systems," Proc. of the IEEE, vol. 95, no. 1, pp. 138-162, 2007.

[21] J. Baillieul and P. Antsaklis, "Control and communication challenges in networked real-time systems," Proc. of the IEEE, vol. 95, no. 1, pp. 928, 2007.

[22] P. Garcia, P. Castillo, R. Lozano, and P. Albertos, "Robustness with respect to delay uncertainties of a predictor-observer based discrete-time controller," Proc. of the 45th IEEE Conf. on Decision and Control, pp. 199-204, 2006.

[23] C. Lien, "Robust observer-based control of systems with state perturbations via LMI approach," IEEE Trans. Autom. Control, vol. 49, no. 8, pp. 13651370, 2004.

[24] A. Sala, "Computer control under time-varying sampling period: An LMI gridding approach," Automatica, vol. 41, no. 12, pp. 2077-2082, Dec. 2005.

[25] J. Li, Q. Zhang, Y. Wang, and M. Cai, " $H \infty$ control of networked control systems with packet disordering," IET Control Theory Appl., vol. 3, no. 11, pp. 1463-1475, March 2009.

[26] Y. Zhao, G. Liu, and D. Rees, "Improved predictive control approach to networked control systems," IET Control Theory Appl., vol. 2, no. 8, pp. 675681, Aug. 2008.

[27] K. Astrom, "Event based control," Analysis and Design of Nonlinear Control Systems, pp. 127-147, 2007.

[28] A. Cuenca, P. García, K. Arzén, and P. Albertos, “A predictor-observer for a networked control system with time-varying delays and non-uniform sampling," Proc. Eur. Control Conf., pp. 946-951, 2009.

[29] J. Xiong and J. Lam, "Stabilization of linear systems over networks with bounded packet loss,"
Automatica, vol. 43, no. 1, pp. 80-87, 2007.

[30] H. Song, L. Yu, and A. Liu, " $H \infty$ filtering for network-based systems with communication constraints and packet dropouts," Proc. of the 7th Asian Control Conf., pp. 220-225, 2009.

[31] P. Garcia, A. Gonzalez, P. Castillo, R. Lozano, and P. Albertos, "Robustness of a discrete-time predictor-based controller for time-vaying measurement delay," Proc. of the 9th IFAC Workshop on Time Delay Systems, 2010.

[32] J. Sturm, "Using SeDuMi 1.02, a MATLAB toolbox for optimization over symmetric cones," Optimization methods and software, vol. 11, no. 1, pp. 625-653, 1999.

[33] T. Henningsson and K. Astrom, "Log-concave observers," Proc. of the $17^{\text {th }}$ Int. Symp. on Mathematical Theory of Networks and Systems, pp. 2163-2170, 2006.

[34] D. Davison and E. Hwang, "Automating radiotherapy cancer treatment: Use of multirate observer-based control," Proc. of American Control Conf., vol. 2, pp.1194-1199, 2003.

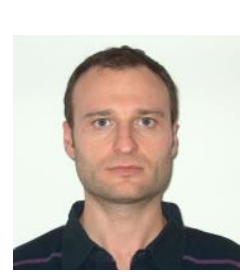

Ángel Cuenca received his M.Sc. degree in Computer Science in 1998 and his $\mathrm{Ph}$. D. in Control Engineering in 2004, from the Technical University of Valencia. Assistant Profesor since 2000, he is with the Systems Engineering and Control Dept. at the Technical University of Valencia, Spain. He has co-authored over 30 papers in congress communications and journals. His research interests include multi-rate control systems, networked control systems, and event-based control systems.

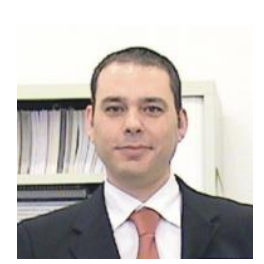

Pedro García was born in Requena, Spain. In 2007 he obtained his Ph.D. in Control Systems and Industrial Computing from Technical University of Valencia, Spain. He is currently Assistant Profesor of Automatic Control at the Technical University of Valencia. $\mathrm{He}$ has co-authored more than ten papers in middle or top impact journals. His research interests are within the broad area of time delay systems and real time control.

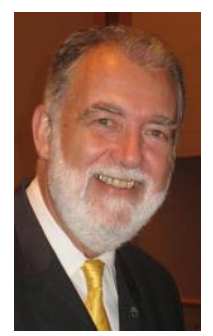

Pedro Albertos, past president of IFAC (the International Federation of Automatic Control) in 1999-2002, IFAC Fellow, IFAC Advisor and Senior Member of IEEE, is a world recognized expert in real-time control, leading several projects in the field. Full Professor since 1975, he is currently at Systems Engineering and Control Dept. UPV, Spain. He is Doctor HonorisCausa from Oulu University (Finland) and Bucharest Polytechnic (Rumania). Invited Professor in more than 20 Universities, he delivered seminars in more than 30 universities and research centres. Authored over 300 papers, book chapters and congress communications, co-editor of 7 books and coauthor of "Multivariable Control Systems" (Springer 2004) and 
"Feedback and Control for Everyone" (Springer 2010), he is also associated editor of Control Engineering Practice and Automatica and Editor in Chief of the Spanish journal RIAI. His research interest includes multivariable control and nonconventional sampling control systems, with focus on time delays and multirate sampling patterns.

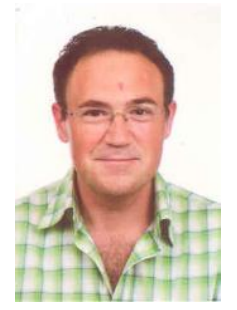

Julián Salt received his M.Sc. Degree in Industrial Engineering in 1986 and his Ph.D. in Control Engineering in 1992, from the Technical University of Valencia. His current position is as full Professor at the Technical University of Valencia. Recently he was Head of the Department of Systems Engineering and Control at the Technical University of Valencia. He has been Director of nine PhD theses and coauthor of about 70 papers in journals and technical meetings. His research interests include non-conventionally sampled control and networked control systems. 